\title{
Discovery of Signature Cyclic Immonium Ion for Lactyllysine Reveals Widespread and Functional Lactylation on Non-histone Proteins
}

\author{
Hui Ye ( $\nabla$ cpuyehui@cpu.edu.cn ) \\ China Pharmaceutical University https://orcid.org/0000-0002-7416-1558 \\ Ning Wan \\ China Pharmaceutical University \\ Nian Wang \\ China Pharmaceutical University \\ Ying Kong \\ China Pharmaceutical University \\ Xinmiao Wang \\ China Pharmaceutical University \\ Dexiang Wang \\ China Pharmaceutical University \\ Siqin Yu \\ China Pharmaceutical University \\ Wenjie Lu \\ China Pharmaceutical University \\ Yinxue Zhu \\ China Pharmaceutical University \\ Chenxi Yang \\ Southeast University \\ Chang Shao \\ China Pharmaceutical University \\ Runbin Sun \\ China Pharmaceutical University

\section{Nanxi Wang} \\ University of California, San Francisco \\ Haiping Hao \\ China Pharmaceutical University https://orcid.org/0000-0003-2522-7546
}

\section{Article}

Keywords: lactylation, histones, biochemistry

Posted Date: September 24th, 2021

DOI: https://doi.org/10.21203/rs.3.rs-916390/v1

License: (1) This work is licensed under a Creative Commons Attribution 4.0 International License. Read Full License 


\section{Abstract}

Lactylation is a new modification discovered on histones. However, whether it can be installed on non-histone proteins remains unclear. Here we report the formation of a signature cyclic immonium ion of lactyllysine, together with the characteristically changed chromatographic behavior, enabling confident protein lactylation assignment by mass spectrometry. This identification strategy was confirmed by affinity-enriched lactylation proteome and revealed lactylation on nuclear non-histone proteins such as nucleolin. Subsequent exploitation of the approach to mining unenriched, deep proteome resources unveiled an understudied lactylation landscape. From the draft map of the Human Proteome, we identified widespread lactylation on DHRS7 among human tissues, and demonstrated site-directed mutagenesis of the lactylated site affects previously unannotated proteinaceous association. Additionally, the Meltome Atlas showed lactylation frequently occurs on glycolytic enzymes and concomitantly induces thermal stability changes on carrier enzymes. Collectively, the identified signatures of protein lactylation enable confident assignment and allow for the discovery of lactylation proteome expanding beyond histones, representing a step to further understand how lactylation governs cells.

\section{Introduction}

The recently discovered lactylation is a post-translational modification (PTM) derived from glycolysis-produced lactate and initially discovered on the lysine residues of histones in cultured human cells. Subsequent functional interrogation of this modification reveals that histone lactylation acts as a new epigenetic mark that drives M1 macrophages to turn on M2 gene expression and enacts polarization in mouse bone marrow-derived macrophages (BMDMs) ${ }^{1}$. Furthermore, lactylated histone has also been noted to activate pluripotency gene expression and promote reprogramming of mouse embryonic fibroblasts (MEFs) into induced pluripotent stem cells (iPSCs) ${ }^{2}$. Despite these pioneering research revealing the physiological significance of lactylation in controlling gene expressions through histones, we are intrigued by whether lactylation is a widespread PTM that is also capable of modifying non-histone proteins in human cells as classic acylation modifications $\mathrm{do}^{3,4}$ and can concomitantly instruct structural and functional changes on the target proteins bearing lactylation. Answer to this open question is anticipated to provide new perspectives regarding how lactate dictates a plethora of biological events in distinct human cell communities under diverse pathological conditions exemplified by cancers and autoimmune diseases. Intriguingly, cues of the vast expanse of the biological space of lactylation have been provided by recent global lactylation proteome mapped from non-mammalian species including a plant fungal pathogen Botrytis cinerea ${ }^{5}$ and rice Oryza sativa ${ }^{6}$.

Given so little we currently know about the proteins subjected to lactylation in human cells and tissues, the ability to make confident lactylation assignment is of paramount importance. Conventionally, the principle of identifying protein PTM by mass spectrometry (MS) is that the modified peptide is supposed to confer a fixed mass shift dependent on the specific types of PTM on both the peptide precursors and MS/MS fragment ions ${ }^{7}$. Nevertheless, false positive PTM matches assigned based on this rationale through search engines are reported to be severely underestimated and thus make confident PTM assignment challenging especially for unenriched proteome data ${ }^{8,9}$. The exploitation of signature MS/MS ions to validate the presence of PTMs has thus become a classic practice when handling large-scale proteomics datasets ${ }^{10}$. Exemplified by glycoproteomics analysis, oxonium ions efficiently signify glycosylation and aid to distinguish between certain glycan moieties ${ }^{11}$. In phosphoproteomics, neutral loss of $\mathrm{H}_{3} \mathrm{PO}_{4}$ at $97.98 \mathrm{Da}$ is a diagnostic marker ion of phosphorylated serine and threonine (pSer, pThr) ${ }^{12,13}$. Remarkably, a clear immonium ion of phosphotyrosine (pTyr) at $m / z 216.0426$ in tandem MS spectra following the higher-energy C-trap dissociation (HCD) fragmentation pinpoints the modification, and differentiates it from pSer and pThr ${ }^{14}$. Consistently, acetylation at lysine residues can generate a facile acetylysine immonium ion at $m / z 143.1179$ and subsequently produce a secondary immonium ion at $m / z 126.0913^{15}$, which serves as a substitute for the former with improved specificity albeit still suffers from low sensitivity in acetylation recognition ${ }^{16}$. Besides these prevalent PTMs, diagnostic value of immonium ions culminates in confident identification of novel PTMs such as phosphohistidine ${ }^{17}$, arginine rhamnosylation ${ }^{18}$ and lysine hydroxypimelylation ${ }^{19}$ that have all been firstly and only discovered in bacteria. Collectively, these findings elicit us to seek for reliable signature ions for lactylation given the depth current proteomics technology can reach even without affinity enrichment.

Here we employed non-enzymatic chemical labeling approaches to introduce lactylation on lysine residues of model peptides and proteins ${ }^{20,21}$. Tandem MS spectra of lactylated peptides acquired on different MS platforms unequivocally led us to note an intense peak corresponding to the "cyclic" immonium (cyclm) ion of lactyllysine that is generated from the "linear" immonium (linlm) ion upon the loss of $\mathrm{NH}_{3}$. The cyclm ion provides superior sensitivity and specificity as a lactylation marker than the lin Im ion. Ion mobility further recorded their conformotypic information and is useful to distinguish these ions from confounding MS/MS ions and improves the specificity in signifying lactylation. Together with the delayed elution on reversed phase columns due to enhanced hydrophobicity conferred by lactylation, we comprehensively defined the diagnostic behaviors of protein lactylation on LC-MS/MS. Exploitation of these characteristics to analyze affinity-enriched lactylation proteome of cultured human cells ${ }^{1}$ demonstrated the diagnostic value of the cyclm ion and allowed us to identify non-histone nuclear proteins exemplified by nucleoin (NCL) whose lactylation levels were tightly regulated by glycolytic flux, phenocopying the responsiveness of histone. We further applied the signature ion to mining lactylation proteome from unenriched, whole human proteome resources. Intriguingly, analysis of the draft map of the Human Proteome ${ }^{22}$ pinpoints widespread lactylation on short-chain dehydrogenase/reductase (SDR) family member 7 (DHRS7) in multiple human tissues, whereas site-directed mutagenesis of this site prompted us to note previously unannotated proteinaceous association. Another large-scale proteome data resource, the Meltome Atlas ${ }^{23}$, reveals that glycolytic enzymes are most heavily lactylated in examined human cells. Subsequent topological analysis indicates lactylation events occur on lysines of active sites and predicts them to be enzymatic activity modulators. In support of this implication, we found certain carrier enzymes exhibited thermal stability shifts upon lactylation, suggesting this modification is capable of influencing the biochemical properties of substrate proteins and hence their structures and functions ${ }^{24}$. Together, the ability to confidently identify lysine lactylation empowers mapping the landscape of human lactylation proteome and fosters ensuing investigation into the regulatory mechanisms of lactylation when our understanding to this newly discovered PTM is still during its infancy. 


\section{Results}

\section{Discovery of a signature cyclic immonium ion from lysine lactylated peptides}

Driven by the urge to discover unknown lactylation substrate proteins, we sought to identify unique features of lactyllysine peptide carriers on LC-MS/MS. We first chemically introduced lactylation onto lysines of model peptides LVFFKA and NKGAll (Fig. 1a, Fig. S1a). Successful derivatization, both the L- and Dlactylation ${ }^{1,21}$, was confirmed by a mass shift of $72.02 \mathrm{Da}$ on the peptide precursors and the collision-induced dissociation (CID)-generated MS/MS fragment ions that carry the modification using a Q/TOF (Fig. 1b-c, Fig. S1b-c). Further scrutinization of the low-mass region elicited us to note a clear ion at $m / z 173.129$ corresponding to the linlm ion of lactyllysine (Fig. 1c). Notably, this ion is conducive to lose $\mathrm{NH}_{3}$ (Fig. $1 \mathbf{d}$ ), and is thus accompanied by the generation of a cyclic form of immonium (cyclm) ion at $\mathrm{m} / \mathrm{z} 156.103$ that exhibited significantly higher intensity than the linlm ion (Fig. 1c, Fig. S1c). Consistently high intensity of the cyclm ion over the lin Im ion, both yielded by lactyllysine, was confirmed by screening the MS/MS spectra of two lactylated model peptides acquired at distinct collision energy (CE) spanning a wide range (Fig. S1d-e).

\section{Cyclic immonium ion of lactyllysine signifies lactylation}

Then, we sought to investigate the diagnostic value of the cyclm ion to signify the occurrence of lysine lactylation using the human recombinant ENO1 as a model protein. We employed a mild, nondenaturing chemical reaction by incubating ENO1 with LGSH ${ }^{21}$ (Fig. S1a), and performed bottom-up analysis on the lactylated protein using an Orbitrap. Subsequent mass shift-based database search and manual inspection led us to identify 28 lactylated lysines on ENO1, which corresponds to 100 lactylated peptides and 357 PSMs (Fig. 1e). We also conducted the analysis on a Q/TOF (Fig. S2a). Interesting, regardless of the instrument platforms and database search engines we tested, analysis of the MS/MS spectra of the in vitro lactylated ENO1 peptides demonstrated that lactyllysine carriers rather than non-lactylated peptides are prone to produce the cyclm ion in a greater frequency and at exceedingly higher intensity compared to the linlm ion (Fig. 1f-g, Fig. S2b-d), suggesting the sensitivity and specificity of the cyclm ion for confident lactylation assignment. In agreement, receiver operating characteristic (ROC) curves verified that the AUC value of using the presence of cyclm ion as the marker of lactylation reached 0.91 , whereas AUC of using the linlm ion as lactylation marker was only 0.70 (Fig. 1h). Collectively, using in vitro lactylated peptides we propose that the cyclm ion is a promising marker for lactylation assignment.

Meanwhile, we found the non-specific nature of the linIm ion in signifying lactyllysine can be attributed to the yield of isobaric MS/MS fragment ions from peptides with N-terminal sequences of LS/SL, IS/SI, TV/VT (Fig. S3a). In comparison, interfering MS/MS ion for the cyclm ion are rare. Given ion mobility is powerful in differentiating isobaric molecules based on gas-phase conformations ${ }^{25,26,27}$, we subsequently tested whether ion mobility can accurately measure the collision cross section (CCS) values of the signature ions and thus enable distinguishing the linlm ion from interfering isomeric MS/MS ions. We employed a travelling wave ion mobility mass spectrometer (TWIMS) to induce MS/MS fragmentation for three lactylated peptides and then analyzed the arrival time distributions (ATDs) for the produced MS/MS ions (Fig. S3b). Indeed, the detected ATDs of the diagnostic cyclm ions produced from LVFFK ${ }^{\text {Lac }}$ A, NK ${ }^{\text {Lac }}$ GAll and FNK ${ }^{\text {Lac }}$ and hence the corresponding CCS values all resembled (Fig. S3b). Moreover, TWIMS enabled us to unambiguously discern the linlm ion at $m / z$ 173.1290 from the isobaric MS/MS ion, $\mathrm{a}_{2}$ ion of dipeptidyl Ser-lle at $m / z 173.1290$, by distinct ATDs (Fig. S3c). Consequently, although current MS proteomics workflow is still unamenable to record the CCS value of all the MS/MS fragment ions produced from peptide precursors on-the-fly and thus resolve confounding peaks for the marker ions of lactyllysine via ion mobility, we anticipate this analytical challenge will be overcome by future advancement of instrumentation and endow the lactyllysine diagnostic ions with even greater specificity and hence increased diagnostic value for lactylation assignment.

\section{Lysine lactylation modifies peptide chromatographic behavior}

Besides the signature cyclm ion, we postulate that the biochemical properties of lactylation peptide carriers are also affected by this modification. We thus compiled the assigned lactylated ENO-digested peptides and the unmodified peptides of identical sequences based on shotgun proteomics data (Fig. S2a and Table S1). Pairwise analysis of these peptides suggests a marked increase in peptide retention time conferred by lactylation (Fig. 2a-b, Fig. S4a and Table S1). Further, we analyzed the chromatographic behaviors of lactylated vs. unmodified peak pairs based on bottom-up proteomics data of lactylated ENO1 collected on different analytical instruments, and verified this finding with 122 peptide pairs detected by timsTOF and 97 pairs by an Orbitrap (Fig. 2c, Fig. S4bd). Consistently, the lactylation-induced peptide retention time shift was substantiated by bottom-up analysis of additional in vitro lactylated BSA and lysozyme (Fig. 2c). Distribution of the retention time shift of lactylated peptides was summarized (Fig. 2d). Then, as we have analyzed the model protein digests with trapped ion mobility spectrometry (TIMS) using a parallel accumulation-serial fragmentation (PASEF) approach ${ }^{28}$, the recorded CCS values of lactylated vs. unmodified peptide precursors pairs were subjected to comparison. Nevertheless, this pilot study identifies no significant changes in ion mobility behaviors $\left(1 / \mathrm{K}_{0}\right)$ being brought to the examined peptide carriers by lactylation, posing a stark contrast to altered chromatographic behaviors (Fig. $2 \mathrm{e}$ ). Collectively, retention time increase on a reversed-phase column is believed to serve as an additional indicator of lactylation and further eliminate false positive matches in combination with the cyclm ion.

\section{Benchmarking diagnostic cyclm ion with affinity enriched-lactylation proteome data}

We next asked whether the signature cyclm and linlm ions of lactylated peptides discovered on model peptides and proteins also apply to complex human cellular proteome. We first validated the origin of the cyclm and linlm ion by analyzing the metabolically labeled proteome data that was collected from MCF-7 cells cultured with isotopically labeled glucose $\left({ }^{13} \mathrm{C}_{6}\right.$-glucose). Theoretically, ${ }^{13} \mathrm{C}_{6}$-glucose is endogenously metabolized to ${ }^{13} \mathrm{C}_{3}$-lactate and is expected to induce a mass shift of $75.0312 \mathrm{Da}$ for isotopically lactylated peptides, which accords to $\triangle \mathrm{m}=3.0101 \mathrm{Da}$ compared to light lactylation peptides when cells were cultured in ${ }^{12} \mathrm{C}_{6}$-glucose (Fig. 3a). Such mass shift was confirmed for isotopically lactylated peptide precursors and also for the cyclm and linlm ions that both inherited the three-carbon units from lactate and hence delivered $m / z 159.1121$ and $m / z 176.1381$, respectively (Fig. 3a). Meanwhile, the ensuing analysis of an open-access quantitative proteome dataset collected from MCF-7 cells labeled by amino acids in cell culture (SILAC) and enriched by pan- 
lactylation antibody further led us to note that the signature cyclm and linlm ions also inherited the heavy isotopes introduced by lysine by comparing the light and heavy group of SILAC proteome. Specifically, in the heavy-labeled group the linlm ion of lactylation peptide inherited ${ }^{13} \mathrm{C}_{6}$ and ${ }^{15} \mathrm{~N}_{2}$ and increased its mass to $\mathrm{m} / \mathrm{z}$ 180.1391, whereas the cyclm ion lost a $\mathrm{NH}_{3}$ and inherited ${ }^{13} \mathrm{C}_{6}$ and ${ }^{15} \mathrm{~N}$ as evidenced by its increase to $\mathrm{m} / \mathrm{z} 162.1154$ (Fig. 3b). Combinatorially, we substantiated the cyclm and lin Im ions of lyactyllysine inherit the isotopically labeled C derived from lactate and lysine as well as $\mathrm{N}$ from lysine, supporting the proposed production pathway (Fig. 1d).

Confirmation of lactyllysine as the origin of the detected cyclm and linIm ions in tandem MS spectra thus led us to appraise the production efficiency of both ions from lactylation proteome of cultured human cells. We analyzed two publicly accessible, SILAC-processed and affinity enriched proteome datasets collected from MCF-7 cells in response to rotenone and DCA intervention ${ }^{1}$ (Fig. 3c, Table S2). Excitingly, after database searching against the two datasets, we found the cyclm ion was detected in lactylated peptides at a percentage as high as $89 \%$ and $90 \%$ whereas the linlm ion displayed a lowered percentage at $76 \%$ and $79 \%$ (Fig. 3c). Thus, we corroborated the high production rate of cyclm ion from lactylated peptides using affinity-enriched lactylation cellular proteomics data (Fig. 3c). Then, we investigated whether the cyclm ion is produced at high intensity in the examined affinity-enriched lactylation proteome datasets, and found the relative ion abundance of the cyclm ion in MS/MS spectra of lactylated peptides reached as high as $39.74 \%$ and $44.17 \%$ from the two datasets (Fig. 3d, Table S2), supporting the cyclm ion as a sensitive marker in signifying lactylation from complex cellular proteome.

\section{Uncover non-histone nuclear proteins as lactylation substrates from affinity-enriched human cell proteome}

Upon validating the usefulness of the cyclm ion in lactylation site assignment from the affinity proteomics data, we are curious whether lactylation modifies human non-histone proteins and believe this knowledge holds the key to elucidate unexplored regulatory functions of lactylation besides being a histone mark. We used the presence of the cyclm ion to filter all database searching-identified lactylation peptides from the affinity proteomics data. As a result, we retrieved 97 lactylated peptides and 84 lactylation sites belonging to 35 human non-histone proteins and 16 histones, whereas 9 lactylation site and 11 lactylation peptides lacking the cyclm ion in MS/MS spectra were removed as false positive matches. Driven by the observation that lactylation does not exclusively modify human histones, we proposed to infer the regulatory roles of lactylation on non-histone proteins by conducting gene ontology (GO) analysis including cellular component (CC), molecular function (MF) and biological processes (BP) on the newly identified lactylated proteins. Since the revisited proteomics data was collected following a histone extraction protocol, $\mathrm{CC}$ analysis shows that the identified lactylated proteins were mostly distributed in chromatin and nucleus (Fig. S5a). In agreement, MFs of the lactylated proteins were mainly classified as RNA and DNA binding (Fig. S5a). Noteworthy, we found lactylation can be installed on non-histone transcriptional regulators including High mobility group protein HMG-I/HMG-Y (HMGA1), General transcription factor II-I (GTF2I) and Zinc finger protein 706 (ZNP706) (Fig. S5b, Table S2), warranting future exploration of the functional readouts of lactylation on proteins of this class. Next, since lysine residues is a prevalent residue that bears other regulatory PTMs including ubiquitination, methylation, acetylation and sumoylation, we performed PTM category analysis for all the identified lactylated lysines and found they are prone to carry PTMs such as acetylation and ubiquitination compared to all quantified lysine residues (Fig. S5c), suggesting the tendency of PTM crosstalk occurring on the lactylated proteins.

Next, we queried whether the lactylation levels of these identified substrate proteins can respond to glycolysis and hence phenocopy histone lactylation ${ }^{1}$. Specifically, activated glycolysis was achieved through the inhibition of oxidative phosphorylation by rotenone while the glycolysis flux was inhibited by sodium dichloroacetate (DCA) treatment. By performing quantitative analysis of the lactylated proteome, we confirmed that the abundance levels of the lactylated peptides were mostly increased by rotenone and lowered by DCA, in concert with the abundance changes of lactate (Fig. 3e, Table S2). Although we noted that the lactylation sites sensitively responding to glycolytic activation and inhibition are not fully overlapped (Fig. 3f-g, Fig. S5d, Table S2), comparative analysis of the dynamic lactylation proteome shows that certain non-histone lactylation sites such as K102 and K116 on human nucleolin (NCL, Fig. S5e) were tightly controlled by both rotenone and DCA treatment (Relative abundance change>1.5). Such dynamic responses are in concert with a previously reported epigenetic mark, K9 lactylation on Histone H4 (Fig. 3f-g). Notably, NCL is an abundant nucleolar protein that has been demonstrated to be involved in modulating mRNA turnover and transcription, pre-rRNA transcription and processing, nucleolar chromatin remodeling and ribosomal assembly. Thus, our discovery on NCL lactylation bolsters an intriguing hypothesis that lactylation may control transcription of downstream target genes through diverse recipients expanding beyond histones in human cells.

\section{Diagnostic ion reveals widespread DHRS7 lactylation from the draft map of the Human Proteome}

Next, we wondered whether the signature cyclm ion benchmarked by the analysis of affinity-enriched lactylation proteome is useful for globally mapping lactylation proteins from large-scale, unenriched human proteome resources. Thus, we first performed database searching against lysine lactylation from the draft map of the Human Proteome 22 , which delivered multiple hits. However, most of the identified lactylated peptides failed to produce the diagnostic cyclm ion and are thus believed as false positive matches (Fig. 4a). For those indeed yielding cyclm ions in MS/MS spectra, we can make the assignment with very high confidence. The increased hydrophobicity and hence delayed elution time further aid to confirm the assigned lactylation sites (Fig. S6a), recapitulating the observation we made using model proteins (Fig. 2a-d). Among the assigned true lactylation carriers (Fig. 4a), an understudied enzyme DHRS7 was repetitively identified to carry lactylated K321 in human tissues including liver, retina, spinal cord, testis, ovary and prostate (Fig. 4b). We confirmed this lactylation site can be identified by database searching using three different search engines (Fig. S6b, Table S3). Moreover, the cyclm ion consistently showed significant abundance in the MS/MS spectra of the lactylated DHRS7 peptide regardless of the tissue origins from which the proteomics data was collected (Fig. 4a, Fig. S6c). Of note, the lactylation sites and protein substrates that we identified from the draft map of the Human Proteome using different search engines actually varied (Fig. S6d, Table S3), reiterating the importance of establishing a gold standard for confident lactylation assignment.

DHRS7 belongs to the SDR superfamily that usually catalyze NAD $(\mathrm{P})(\mathrm{H})$-dependent reactions with a large array of substrates, including steroid hormones, prostaglandins, retinoids, lipids and xenobiotics ${ }^{29,30}$. However, DHRS7 and its functional interplay remains understudied, nonetheless the discovered lactylation site K321. Predicted protein structure of DHRS7 revealed that K321 is located in an \$-helix in proximity to the protein C-terminus (Fig. 4c). Its 
functional importance is implied by the previously reported methylation and ubiquitination on the identical residue (Fig. 4c). The evolutionary conservation of K321 across eukaryotic phylogeny also support the likelihood of this residue and hence the installed lactylation to be functionally important for DHRS7 (Fig. 4d).

\section{Exploring regulatory mechanism and biological readouts of DHRS7 lactylation}

Before we set out to assess how this modification may perturb the function of DHRS7, we must firstly validate the lactylation on K321 of DHRS7. Therefore, we overexpressed His-tagged and FLAG-tagged DHRS7 in HEK293T cells (Fig. S6e). Indeed, we identified lactylated K321-containing peptides from the lysed cells by database searching, and further confirmed the assignment by the presence of the cyclm ion at $\mathrm{m} / \mathrm{z} 156.1025$ in the resultant MS/MS spectra (Fig. 5ab) along with their significantly delayed elution on a $\mathrm{C}_{18}$ column compared to the unmodified counterparts (Fig. 5c). The substantiated lactylation on K321 of DHRS7 thus prioritized our investigation of this residue as a functional hotspot. Since DHRS7 is abundant in human prostate tissue and pronouncedly upregulated in prostate adenocarcinoma patients (Fig. S6f), we overexpressed the DHRS7 plasmid in a human prostate cancer cell line PC3. Surprisingly, lactylation on K321 of DHRS7 as we observed in HEK293T cells was dampened in DHRS7-overexpressed PC3 cells and the unmodified DHRS7 became the bulk proteoform, phenocopying a nonlactylated K321A mutant DHRS7-transfected cells (Fig. 5d). Hence, the distinct lactylation statuses on K321 in HEK293T and PC3 cells stimulate the interrogation of the expression levels of potential lactylation "writers" and "erasers" in these two cell lines, respectively. Comparative proteomics analysis of the HEK293T and PC3 cells has shown that, among the currently verified delactylases, HDAC3 was detected in PC3 cells yet absent in the detected HEK293T proteome (Fig. 5e-f). Considering a recent biochemical study that demonstrated HDAC3 as an efficient lactylation eraser ${ }^{31}$, it is likely that lactylation is maintained on K321 in HEK293T cells while removed in PC3 cells due to the cell-type specific, differential delactylase levels. Collectively, our in vitro findings laid a foundation for future systematic screening of lactylation writers and erasers responsible for DHRS7 and possibly for other substrate proteins, holding the potential to resolve the puzzle of the regulatory enzymatic systems for lactylation in mammalian cells.

Lastly, given lactylation together with previously recorded methylation and ubiquitination all being installed on K321, we hypothesized that this residue is important for DHRS7 and may affect its structure, function, protein engagement or the transduced signaling network and ultimately certain phenotypes of cells. We transiently overexpressed the wild-type (WT) and the nonmodifiable K321A mutant DHRS7 constructs in HEK293T cells and employed shotgun proteomics to globally evaluate the cellular responses (Fig. $\mathbf{5 g}$ ). We found that the K321A point mutation maintained the overall abundance of the global proteome yet caused significant abundance changes for a small subset of proteins including the prosaposin protein (PSAP) (Fig. $\mathbf{5 g}$ ), unveiling a previously unannotated link between DHRS7 and PSAP. A cue of this association is provided by a single cell transcriptomics analysis defining both DHRS7 and PSAP as top signatures of metastasizing cancer cells ${ }^{32}$. Besides proteomics, we further employed metabolomics to interrogate whether lactylated K321 on DHRS7 influence cells' metabolic phenotypes. Untargeted metabolomics analysis of the WT and K321A DHRS7-transfected cells indicated that K321 mutagenesis is sufficient to significantly alter the cellular metabolic profiles as shown by the PCA and OPLS-DA score plots (Fig. S6g) and the heatmap of metabolite abundances (Fig. S6h).

\section{Glycolytic enzymes are heavily lactylated in human cells and carry lactylation that perturbs protein stability}

Current analytical advancement has yielded valuable, unenriched deep human proteome data with PTM information recorded yet waiting to be excavated. Together with the signature cyclm ions enabling true lactylation assignment, we sought to discover more lactylation substrate proteins from the Meltome Atlas $^{23}$, one of the recently shared large-scale data resources that measure thermal stability of the global proteome in multiple model organisms. The ability to determine lactylation site from the Meltome would unveil whether lactylation can influence the thermal profiles of its substrate proteins according to a Hotspot Thermal Profiling (HTP) approach ${ }^{24}$, and hence provide a plausible answer regarding the functionality of human lactylation from a biophysical perspective.

We considered lactylation sites identified by conventional mass shift-based database searching as true on condition that the signature cyclm ion can be retrieved from the MS/MS spectra (Fig. S7a-b), whereas systematic increase in retention time conferred by lactylation further supports the assignment (Fig. S8a). Full searching of lactylation from 14 human cell lines available in the Meltome Atlas led to the discovery of totally 246 lactylated peptides and 231 lactylation sites, whereas enzymes involved in glycolysis, the renowned pathway that produces lactate as its end product, is most heavily lactylated (Fig. 6a, Table S4). Since such enzymes are of high abundance in human cell lines, we must rule out the possibility that the observed high frequency of lactylation on glycolytic enzymes is attributed to their high protein abundances. Thus, we summarized protein abundance ranking for not only the enzymes involved in glycolysis but also those from pentose phosphate pathway (PPP) and tricarboxylic acid (TCA) cycle, the other two major metabolic pathways, in each examined cell line (Fig. 6b). We found that enzymes involved in PPP and TCA cycle vs. glycolysis are of similar abundance, whereas their frequencies of being lactylated are distinct, demonstrating that lactylation preferentially modifies glycolytic enzymes. This finding solicits us to infer the existence of a feedback loop, through which the glycolysis-produced lactate regulates glycolysis through lactylation when the glycolysis pathway is hyperactivated and produces excessive lactate. Another intriguing finding is that pathway enrichment analysis of the identified lactylation proteome reveals heavy lactylation on an alternative energy-producing pathway, fatty acid degradation (Fig. S8b), implying a second route that lactylation takes to control energetics production and govern cell metabolism.

Subsequent sequence motif analysis for the lactylation sites shows a mildly conservative presence of leucine and glutamate residues flanking the identified lactyllysine (Fig. S8c). Next, we analyzed the PTM distribution on the lactylated lysines and found that approximately $82.25 \%$ of them have been reported to bear classically functional PTMs including ubiquitination and acetylation (Fig. S8d). This is exemplified by lactylated K343 on ENO1, which has been previously detected to carry acetylation and ubiquitination, and known to locate in the enzymatic active site ${ }^{33,34,35}$. Thus, we conjecture that lactylation on this critical residue would interrupt the substrate-enzyme interaction and hamper the catalytic activity (Fig. 6c). Notably, among all the identified lactylated human proteins across the examined cell lines, lysines of ENO1 were lactylated in the greatest coverage, reaching the peak level at $23.68 \%$ in colon cancer spheroid cells (Fig. 6c, Fig. S7b). Another example is lactylated K147 shared by highly homologous ALDOA and ALDOC, refreshing our knowledge of this residue as merely an acetylation and ubiquitination carrier ${ }^{33,34}$. Intriguingly, K147 represents the most frequently lactylated residue in the Meltome Atlas based on its 
ubiquitous presence in cell lines including K562, Jurkat, A549, HL60, colon cancer spheroids, HaCaT, HAOEC and HEK293T cells (Fig. S7b). Considering the low human cell line specificity of K147 lactylation, it is tempting to speculate that lactylation would uniformly disturb the enzymatic activity of ALDO and hence inhibit glycolysis given K147 is known to be important for substrate binding ${ }^{36}$. Together, the lactylation distribution on glycolytic enzymes suggests a regulatory mechanism that glycolytic flux can be controlled by redundantly modulated glycolytic enzyme activity via lactylation in human cells.

Besides mapping the lactylation proteome landscape, the Meltome Atlas also allows for discerning functional lactylation hotspots that manage to alter the thermal stability of modified human protein substrates. Indeed, the application of the HTP approach detects that the proteoform of ENO1 carrying lactylated K343 displayed an increased thermal stability when compared to the bulk, unmodified protein in colon cancer spheroids (Fig. 6d). Then, we wondered whether this lactylation-induced change to the intrinsic biophysical property of ENO1 is systematic. Intriguingly, we found the thermal stability shift we observed for lactylated K343 of ENO1 disappeared for the ENO1 proteoform bearing lactylated K326 (Fig. 6d), consistent with previously reported site-specific and unsystematic phosphorylation-induced protein thermal stability change ${ }^{37}$. Another finding is that the lactylation-induced protein thermal stability shift is dependent on cellular millieu. For instance, the lactylated K11 proteoform of the glycolytic enzyme PGK1 shows decreased thermal stability in A549 cells, whereas this trend was dampened in Jurkat cells (Fig. S9a-b). This context-dependent difference is further validated by thermal profiles of lactylated sepiapterin reductase (SPR) (Fig. S9c). As shown in Fig. S9d, thermal stability curves of the lactylated K226 and the unmodified proteoforms indicate that given lactylation induced a subtle yet clear increase in stability in primary human hepatocytes, whereas such deviation was diminished in HepG2 cells. In agreement, the widespread lactylation on K321 of DHRS7 initially discovered in the draft map of the Human Proteome has been verified in the Meltome Atlas datasets, and this modification was found to exert variant effects on DHRS7 thermal stability in K562, Jurkat cells and colon cancer spheroids (Fig. S9e).

\section{Discussion}

Metabolic flux of lactate has been recently traced to the protein pool and led to the discovery of a novel modification, lactylation, on histone lysine residues in human and mice. Despite emerging interests in decoding the functional readouts of histone lactylation, whether lactylation can impose on non-histone proteins and regulate the target proteins structure and function in human cells and tissues remains an open question. The capability to identify lactylation with very high confidence thus provides the opportunity to answer this question. Driven by this urge, here we introduce a straightforward approach enabling confident lactylation identification by LC-MS/MS and present an initial step to unveil a widespread and functional lactylation proteome that remains understudied in human cells and tissues expanding beyond the known substrate, histones. The identification approach requests that, in addition to lactylationinduced mass shift on peptide precursors and fragment ions, a signature cyclm ion of lactyllysine must be detected in corresponding MS/MS spectra.

Actually, we noticed the cyclm ion for lactyllysine almost serendipitously when analyzing the MS/MS spectra of in vitro lactylated model peptides and proteins due to its dominant intensity over the linlm ion in MS/MS spectra. This phenomenon suggests the tendency of the lin Im ion to lose $\mathrm{NH}_{3}$ in the gas phase. In agreement, previous research has also noted that the facile immonium ion of acetyllysine at $\mathrm{m} / \mathrm{z} 143.12$ is conducive to lose $\mathrm{NH}_{3}$ and delivers a peak of increased intensity at $m / z 126.09^{16,19,38}$. Following the analysis of lactylated model peptides, the validity of the cyclm ion in signifying lactylation was then confirmed by its prevalence in the MS/MS spectra of antibody-enriched lactylation proteome. Consequently, we set the presence of cyclm ion as a standard for screening the lactylation sites assigned by conventional target-decoy database searching strategy when unenriched, deep proteomics data resources were queried. This is a compelling endeavor given the deep coverage that current proteomics studies can achieve through technical advancements such as LC separation ${ }^{39}$, ion mobility ${ }^{25}$ and multiple fragmentation methods ${ }^{40}$. Previously, analytical strategy that attempted to identify major PTMs including phosphorylation and acetylation without enrichment has been practiced ${ }^{14}$. Nevertheless, matters are arising when we assume the global false discovery rate (FDR) estimated by the common target-decoy database searching strategy also accurately thresholds the subset of modified peptides when the majority of detected peptides actually do not carry the designated PTM ${ }^{41}$. This could result in severely underestimated false PTM assignments. Indeed, this pitfall has been demonstrated when researchers searched C-terminal amidation that only occurs on glycine against unenriched spectral data, while it turns out that most of the assigned amidated peptides lacked the requisite presence of C-terminal glycine and were thus false-positive matches ${ }^{9}$. Therefore, in order to exploit the ever-expanding proteome resources that has not been subjected to lactylation affinity enrichment while not producing falsely assigned lactylation results, stringent criteria exemplified by the presence of the cyclm ion is of paramount importance for enabling reliable lactylation assignment. Knowledge gained through data mining is anticipated to readily reveal new substrate proteins, associate the dynamic lactylation levels with pathophysiological changes and bridge the gap between the established pleiotropic functions of lactate and our currently limited understanding of lactylation.

Upon the rigorous screening process, mining the publicly accessible resources exemplified by the draft of the human proteome and the Meltome Atlas has driven us to note rich non-histone proteins and pathways subjected to lactylation modification from human cells and tissues. For instance, a relatively understudied enzyme DHRS7 was consistently identified to carry lactylated K321 in clinically collected human samples and cultured human cells. Besides its prevalence, the Meltome Atlas data further indicated that lactylated K321 is a functional hotspot that managed to alter the thermal stability of DHRS7 in K562 cells, and hence solicited us to interrogate the functional importance of this site. Indeed, we found that site-directed mutagenesis of K321 on DHRS7 significantly induced abundance changes for a small subset of proteins such as PSAP, revealing previously unannotated signal transduction between DHRS7 and PSAP and endowing this residue with new functionality.

Analysis of the Meltome Atlas further reveals that, among the examined human cell lines, glycolysis is the most heavily lactylated pathway. Subsequent crystallographic structures pinpoint certain lactylation sites are in proximity to the active sites of modified glycolytic enzymes and may thus limit the target enzymes' accessibility to substrates and hamper the activities. Thus, our finding provides a new angle for understanding how lactate modulates glycolysispreviously, lactate is speculated to control the biosynthetic pathway through non-covalent interactions and inhibition on the pacemaker enzymes known as "end-product inhibition" 42 . 
Noteworthy, in parallel to the discovery of histone lactylation, an alternative lactylation route has been reported in human cells. Methylglyoxal (MGO), a highly reactive glycolytic byproduct, through glutathione (GSH) conjugation forms lactylglutahione (LGSH) and then transfers the lactyl moiety to lysine, imposing Dlactylation on proteins in a non-enzymatic manner ${ }^{21}$. An alkyne-tagged chemical probe mimicking MGO was developed and found to enrich glycolytic enzymes as potential D-lactylation substrates. Their findings agree with our direct identification of lactylation on these enzymes. Furthermore, given two possible lactylation routes exist, we purposely confirmed that the cyclm ion of lactyllysine and the increased hydrophobicity conferred by lactylation apply to both the lactate derived L-lactylation and the MGO-derived enantiomeric D-lactylation (Fig. 1, Fig. S1).

Deep mining the Meltome Atlas solicited us to notice that the changes of melting behaviors induced by lactylation are not always concordant as cell types vary. This is exemplified by the subtle increase of thermal stability of DHRS7 in K562 cells being abolished in the colon cancer spheroids. The distinction implies that the intrinsic property of the carrier protein is not the sole determinant of lactylation-induced thermal stability whereas intracellular environment involving interacting protein partners, as an example, may substantially affect the biophysical consequences. We also noted site-specific thermal profile changes induced by lactylation on K343 vs. K262 of ENO1, prompting us to speculate that lactylation can choose variable routes to differentially regulate the structure and function via modifying distinct sites on an identical target protein.

In summary, with the view of lactate shifted from a glycolytic by-product to metabolic fuel ${ }^{43}$ and now being recognized as a signaling messenger and functional regulator that is intimately associated with human diseases including cancer, sepsis and immune diseases ${ }^{1}$, a confident lactylation identification strategy is anticipated to spur future research into resolving puzzles like how lactate instructs its target cells such as cytotoxic $T$ cells through lactylation, how lactylation is intricately coordinated with other PTMs to regulate identical substrate proteins and whether blunting or stimulating lactylation is of therapeutic value for disease treatment, when biologists are equipped with the proteomics tool presented in this study.

\section{Declarations}

\section{ACKNOWLEDGEMENTS}

This research was supported by the National Natural Science Foundation of China (grants 82173783 to H.Y., grants 81930109,81720108032 to H.H.), the Natural Science Foundation of Jiangsu Province (BK20180079), the Project of State Key Laboratory of Natural Medicines in China Pharmaceutical University (SKLNMZZ202020), the Overseas Expertise Introduction Project for Discipline Innovation (G20582017001 to H.H.), and Sanming Project of Medicine in Shenzhen (SZSM201801060). We thank Dr. Qing Yu from Harvard University and Dr. Baozhen Shan and Wenting Li from PEAKS Studio for useful discussions.

\section{AUTHOR CONTRIBUTIONS}

H.Y. and H.H. conceived the project; N.W. (Ning Wan), N.W. (Nian Wang), H.Y. and H.H. designed the experiments and analyzed the data; N.W. (Ning Wan), N.W. (Nian Wang) and Y.K. performed the proteomics experiments; D.W. established chemical lactylation methods; C.Y. and Y.Z. performed the ion mobility experiments; N.W. (Ning Wan), R.S. and S.Y. contributed to data analysis; N.W. (Nanxi Wang) and W.L. contributed to plasmid construction; X.W. and C.S. contributed to the metabolomics experiments, N.W. (Ning Wan), H.Y. and H.H. wrote the manuscript.

\section{COMPETING INTERESTS}

The authors declare no competing interests.

\section{MATERIALS \& CORRESPONDENCE}

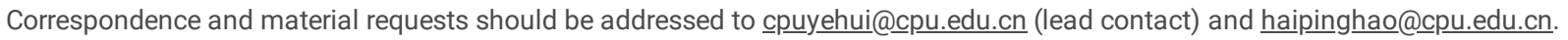

\section{References}

1. Zhang D, Tang Z, Huang H, et. Metabolic regulation of gene expression by histone lactylation. Nature. 2019, 574(7779):575-580.

2. Li L, Chen K, Wang T, et. Glis1 facilitates induction of pluripotency via an epigenome-metabolome- epigenome signalling cascade. Nat Metab. 2020, 2(9):882-892.

3. Fournier M, Orpinell M, Grauffel C, et. KAT2A/KAT2B- targeted acetylome reveals a role for PLK4 acetylation in preventing centrosome amplification. Nat Commun. 2016, 7:13227.

4. Wang Q, Zhang Y, Yang C, et. Acetylation of metabolic enzymes coordinates carbon source utilization and metabolic flux. Science. 2010, 327(5968):10047.

5. Gao M, Zhang N, Liang W. Systematic analysis of lysine lactylation in the plant fungal pathogen Botrytis cinerea. Front Microbiol. $2020,11: 594743$.

6. Meng X, Baine JM, Yan T, Wang S. Comprehensive analysis of lysine lactylation in rice (Oryza sativa) grains. J Agric Food Chem. 2021, $69(29): 8287-8297$.

7. Zolg D, Wilhelm M, Schmidt T, et. ProteomeTools: systematic characterization of 21 post-translational protein modifications by liquid chromatography tandem mass spectrometry (LC-MS/MS) using synthetic peptides. Mol Cell Proteomics. 2018, 17(9):1850-1863.

8. Chalkley R. When target-decoy false discovery rate estimations are inaccurate and how to spot instances. J Proteome Res. 2013, 12(2):1062-4.

9. Anapindi K, Romanova E, Southey B, et. Peptide identifications and false discovery rates using different mass spectrometry platforms. Talanta. 2018, 182:456-463.

10. Kim M, Zhong J, Pandey A. Common errors in mass spectrometry-based analysis of post-translational modifications. Proteomics. 2016, 16(5):700-14. 
11. Morelle W, Michalski J. Analysis of protein glycosylation by mass spectrometry. Nat Protoc. 2007, 2:1585-1602.

12. Bodenmiller B, Mueller L, Mueller M, et. Reproducible isolation of distinct, overlapping segments of the phosphoproteome. Nat Methods. 2007, 4(3):231-7.

13. Schroeder M, Shabanowitz J, Schwartz J, et. A neutral loss activation method for improved phosphopeptide sequence analysis by quadrupole ion trap mass spectrometry. Anal Chem. 2004, 76(13):3590-8.

14. Olsen J, Macek B, Lange O, et. Higher-energy C-trap dissociation for peptide modification analysis. Nat Methods. 2007, 4(9):709-12.

15. Kim J, Kim K, Kwon H, et. Probing lysine acetylation with a modification-specific marker ion using high-performance liquid chromatography/electrospraymass spectrometry with collision-induced dissociation. Anal Chem. 2002, 74(21):5443-9.

16. Trelle $\mathrm{M}$, Jensen $\mathrm{O}$. Utility of immonium ions for assignment of epsilon-N-acetyllysine-containing peptides by tandem mass spectrometry. Anal Chem. 2008, 80(9):3422-30.

17. Potel C, Lin M, Heck A, et. Widespread bacterial protein histidine phosphorylation revealed by mass spectrometry-based proteomics. Nat Methods. 2018, 15(3):187-190.

18. Lassak J, Keilhauer E, Fürst M, et. Arginine-rhamnosylation as new strategy to activate translation elongation factor P. Nat Chem Biol. 2015, 11(4):266-70.

19. Muroski J, Fu J, Nguyen H, et. Leveraging immonium ions for targeting acyl-lysine modifications in proteomic datasets. Proteomics. 2021, 21(34):e2000111.

20. Calderone C, Bumpus S, Kelleher N, et. A ketoreductase domain in the PksJ protein of the bacillaene assembly line carries out both alpha- and beta-ketone reduction during chain growth. Proc Natl Acad Sci U S A. 2008, 105(35):12809-14.

21. Gaffney D, Jennings E, Anderson C, et. Non-enzymatic lysine lactoylation of glycolytic enzymes. Cell Chem Biol. 2020, 27(2):206-213.

22. Kim M, Pinto S, Getnet D, et. A draft map of the human proteome. Nature. 2014, 509(7502):575-81.

23. Jarzab A, Kurzawa N, Hopf T, et. Meltome atlas-thermal proteome stability across the tree of life. Nat Methods. 2020, 17(5):495-503.

24. Huang J, Lee G, Cavanaugh K, et. High throughput discovery of functional protein modifications by hotspot thermal profiling. Nat Methods. 2019, 16(9):894-901.

25. Meier F, Park M, Mann M. Trapped ion mobility spectrometry (TIMS) and parallel accumulation - serial fragmentation (PASEF) in proteomics. Mol Cell Proteomics. 2021, 17:100138.

26. Hines K, May J, McLean J, et. Evaluation of collision cross section calibrants for structural analysis of lipids by traveling wave ion mobility-mass spectrometry. Anal Chem. 2016, 88(14):7329-36.

27. Lu G, Xu X, Li G, et. Subresidue-resolution footprinting of ligand-protein interactions by carbene chemistry and ion mobility-mass spectrometry. Anal Chem. 2020, 92(1):947-956.

28. Meier F, Brunner A, Koch S, et. Online parallel accumulation-serial fragmentation (PASEF) with a novel trapped ion mobility mass spectrometer. Mol Cell Proteomics. 2018, 17(12):2534-2545.

29. Araya S, Kratschmar D, Tsachaki M, et. DHRS7 (SDR34C1) - a new player in the regulation of androgen receptor function by inactivation of $5 a-$ dihydrotestosterone? J Steroid Biochem Mol Biol. 2017, 171:288-295.

30. Zemanová L, Kirubakaran P, Pato I, et. The identification of new substrates of human DHRS7 by molecular modeling and in vitro testing. Int $\mathrm{J}$ Biol Macromol. 2017, 105(Pt 1):171-182.

31. Moreno-Yruela C, Zhang D, Wei W, et. Class I histone deacetylases (HDAC1-3) are histone lysine delactylases. BioRxiv. $2021,436780$.

32. Puram SV, Tirosh I, Parikh AS, et. Single-Cell Transcriptomic Analysis of Primary and Metastatic Tumor Ecosystems in Head and Neck Cancer. Cell. 2017, 171(7):1611-1624.e24.

33. Wu Q, Cheng Z, Zhu J. et. Suberoylanilide hydroxamic acid treatment reveals crosstalks among proteome, ubiquitylome and acetylome in non-small cell lung cancer A549 cell line. Sci Rep. 2015, 5:9520.

34. Akimov V, Barrio-Hernandez I, Hansen S, et. Ubisite approach for comprehensive mapping of lysine and N-terminal ubiquitination sites. Nat Struct Mol Biol. 2018, 25(7):631-640.

35. Moellering R, Cravatt B. Functional lysine modification by an intrinsically reactive primary glycolytic metabolite. Science. 2013, 341(6145):549-53.

36. Hyndman K, Yang C, Jung H, et. Proteomic determination of the lysine acetylome and phosphoproteome in the rat native inner medullary collecting duct. Physiol Genomics. 2018, 50(9):669-679.

37. Potel CM, Kurzawa N, Becher I, Typas A, Mateus A, Savitski MM. Impact of phosphorylation on thermal stability of proteins. Nat Methods. 2021, 18(7):757-759.

38. Annan R, Biemann K. Utility of N-peracetylation of proteins for their structure determination by mass spectrometry. J Am Soc Mass Spectrom. 1993, $4(2): 87-96$.

39. Bian Y, Zheng R, Bayer FP, et. Robust, reproducible and quantitative analysis of thousands of proteomes by micro-flow LC-MS/MS. Nat Commun. 2020, 11(1):157.

40. Frese CK, Altelaar AF, Hennrich ML, et, Mohammed S. Improved peptide identification by targeted fragmentation using CID, HCD and ETD on an LTQOrbitrap Velos. J Proteome Res. 2011, 10(5):2377-88.

41. Chalkley R. When target-decoy false discovery rate estimations are inaccurate and how to spot instances. J Proteome Res. 2013, 12(2):1062-4.

42. Costa Leite T, Da Silva D, Guimarães Coelho R, Zancan P, Sola-Penna M. Lactate favours the dissociation of skeletal muscle 6-phosphofructo-1-kinase tetramers down-regulating the enzyme and muscle glycolysis. Biochem J. 2007, 408(1):123-30.

43. Rabinowitz J, Enerbäck S. Lactate: the ugly duckling of energy metabolism. Nat Metab. 2020, 2(7):566-571. 


\section{a}
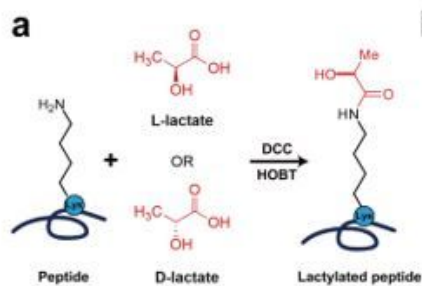

b

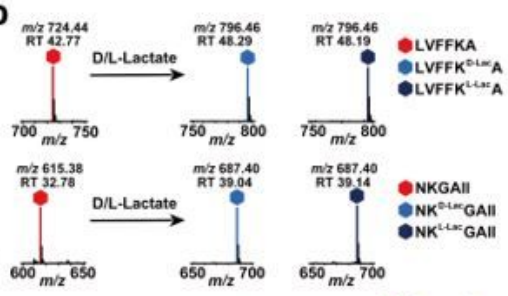

C

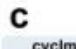

Lactylated peptide $\frac{1}{600 \mathrm{~m} / \mathrm{z}} 6 \mathrm{s0}$

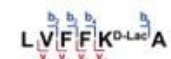
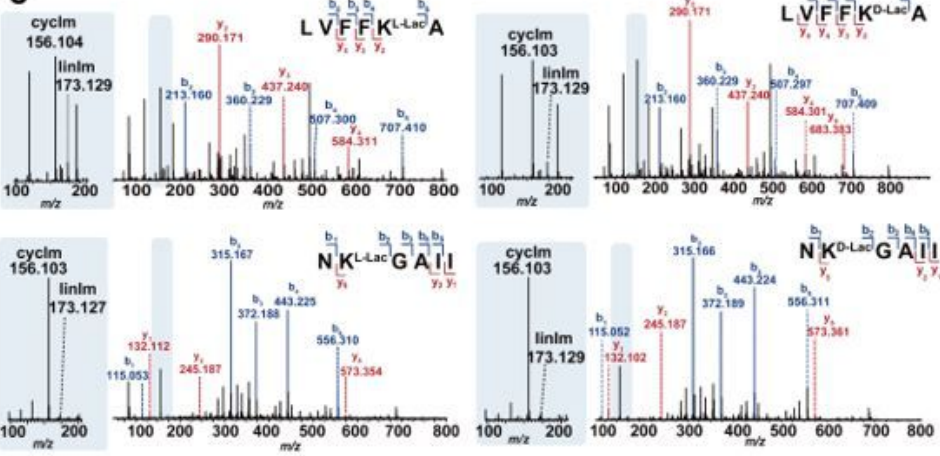

d

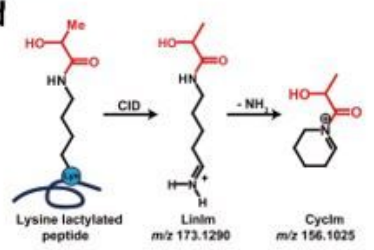

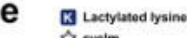
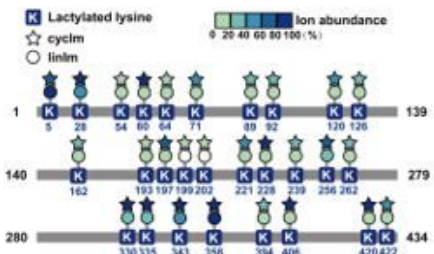

f

g

$h$

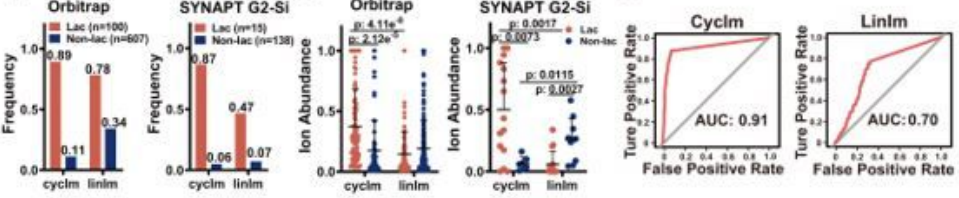

Figure 1

Benchmarking the cyclic immonium ion of lactyllysine to signify protein lactylation by mass spectrometry. (a) Illustration of chemically introduced L/D-lysine lactylation by condensation reaction. DCC, Dicyclohexylcarbodiimide; HOBT, 1-Hydroxybenzotriazole. (b) Mass spectra of L/D-lactylated model peptides. RT, retention time ( $\mathrm{min})$. (c) MS/MS spectra of L/D-lactylated model peptides. (d) Production pathway of the linear (lin Im) and cyclic (cyclm) immonium ions of lactyllysine from lactylated peptides. (e) Illustration of lactylation sites ( $n=28)$ and abundance of the cyclm ion in MS/MS spectra of lactylated ENO1-digested peptides digested from ENO1 ( $n=100)$ based on data collected on an Orbitrap mass spectrometry. (f) Frequency of the lin Im and cyclm ions being detected in lactylated and non-lactylated ENO1 peptides detected by different mass spectrometers. (g) Relative ion abundance of the lin Im and cyclm ions normalized to base peak in lactylated and non-lactylated ENO1 peptides acquired on different mass spectrometers. Data represent mean \pm SD, and statistical significance was determined using unpaired two-tailed t-test. (h) Receiver operating characteristic (ROC) curve analysis of the lin $I m$ and cyclm ion for assigning protein lactylation. AUC, area under the ROC curve. 
a
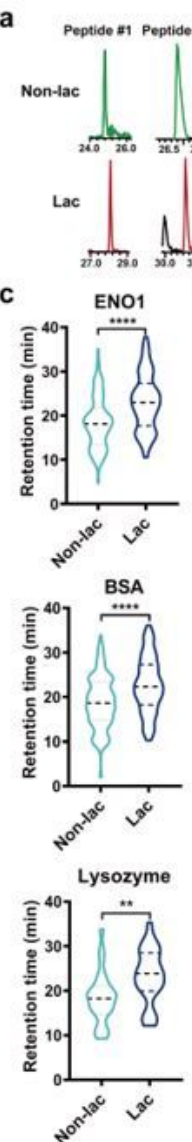
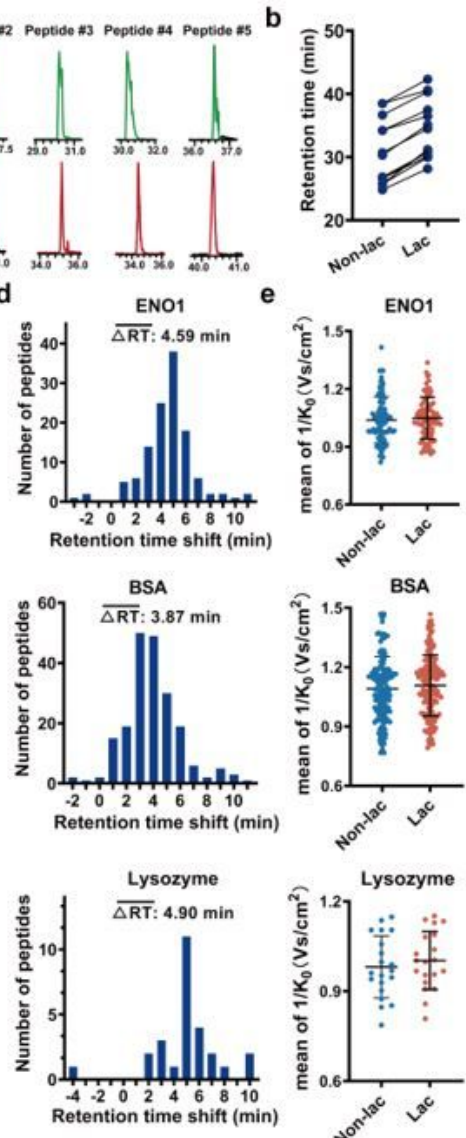

Lysozyme

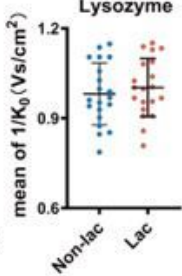

Figure 2

Lysine lactylation-induced changes in peptide chromatographic behaviors. (a) Extracted ion chromatograms (XICs) of lactylated and non-lactylated ENO1digested peptide pairs by LC-MS/MS analysis on a SYNAPT G2 Si Q/TOF. (b) Lactylation-induced retention time shifts of ENO1-digested peptides shown in (a). (c) Retention time distribution of pairwisely matched lactylated and non-lactylated peptides based on bottom-up analysis of in vitro lactylated model proteins on a different Q-TOF, timsTOF. A total of 122, 204 and 27 peptide pairs were summarized for the analyzed ENO1, BSA and lysozyme digests, respectively. Data represent mean $\pm S D$. ${ }^{*} p<0.01, * \star \star * p<0.0001$ by paired two-tailed t-test. (d) Lactylation-induced retention time shifts for the peak pairs summarized in (d). (e) lon mobility values $(1 / \mathrm{K} 0, \mathrm{~V} \cdot \mathrm{s} \mathrm{cm}-2)$ of lactylated and unmodified peptide pairs measured by Trapped lon Mobility Spectrometry (TIMS). 


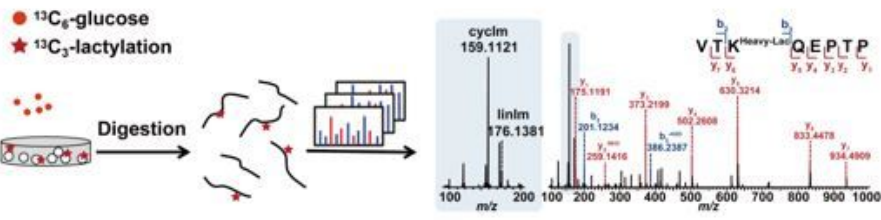

b

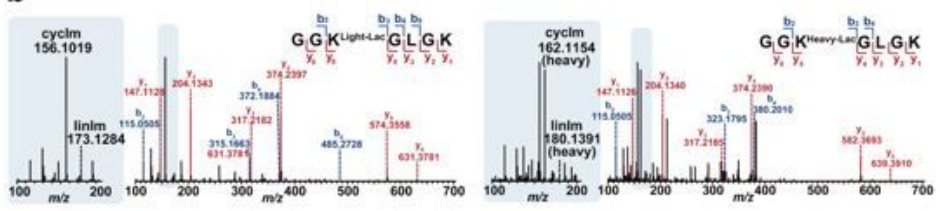

C

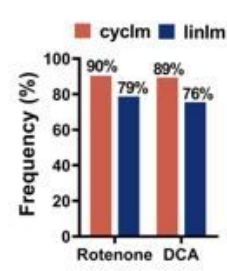

f

d cyclm $\cdot$ linlm

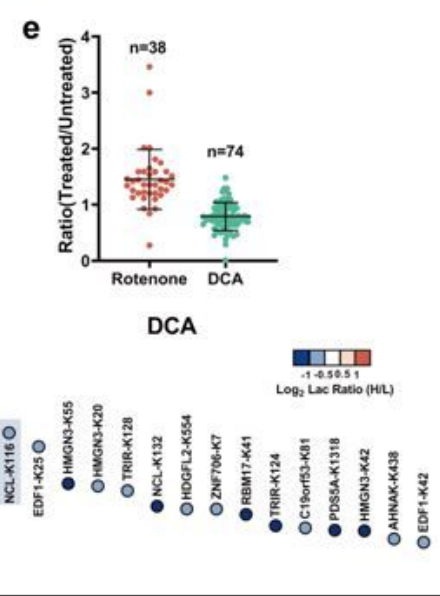

\section{Figure 3}

Diagnostic cyclm ion solicits the discovery of non-histone nuclear proteins from affinity-enriched lactylation proteome of cultured human cells. (a) Workflow of the [13C6]-glucose tracing assay. (b) Representative MS/MS spectra of a lactylated peptide incorporating the 12C614N2-lysine (light group, left panel) and 13C615N2-lysine (heavy group, right panel) in a SILAC-processed proteome dataset. (c) Frequency of the cyclm and linlm ions being detected in MS/MS spectra of lactylation proteome identified from the rotenone and DCA treated MCF-7 cells. A total of 52 and 94 lactylated peptides were identified by conventional database searching from the two SILAC proteomics datasets, respectively. (d) Relative ion abundance of the cyclm and lin Im ions normalized to base peak in each MS/MS spectrum where the signature ions of lactyllysine are retrievable. Data represent mean \pm SD and statistical significance was determined using the unpaired two-tailed t-test. (e) SILAC-based quantitative analysis of lactylated peptides following the rotenone and DCA treatment in MCF7 cells. Each dot in the scatter dot plot represents a lactylated peptide. SILAC ratio was normalized to protein abundance. Data represent mean $\pm S D$. (f-g) SILAC-based quantification of protein lactylation displaying marked changes in response to rotenone (10 nM, $24 \mathrm{~h})(\mathrm{f})$ and DCA (10 mM, $24 \mathrm{~h})$ treatment ( $\mathrm{g})$. Proteomics data used for lactylation mining in Fig. 3 was accessed through the ProteomeXchange Consortium with the dataset identifier PXD014870. 
a

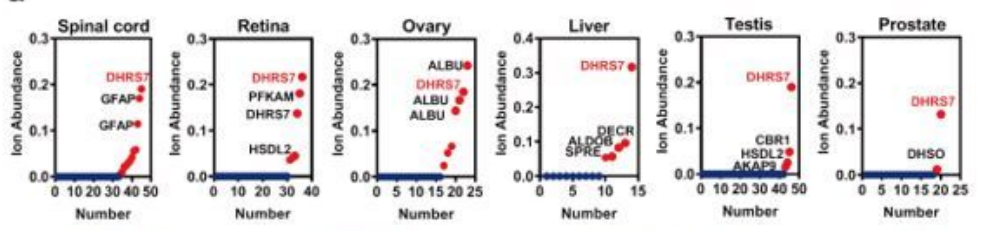

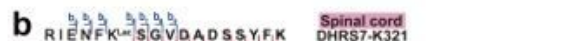
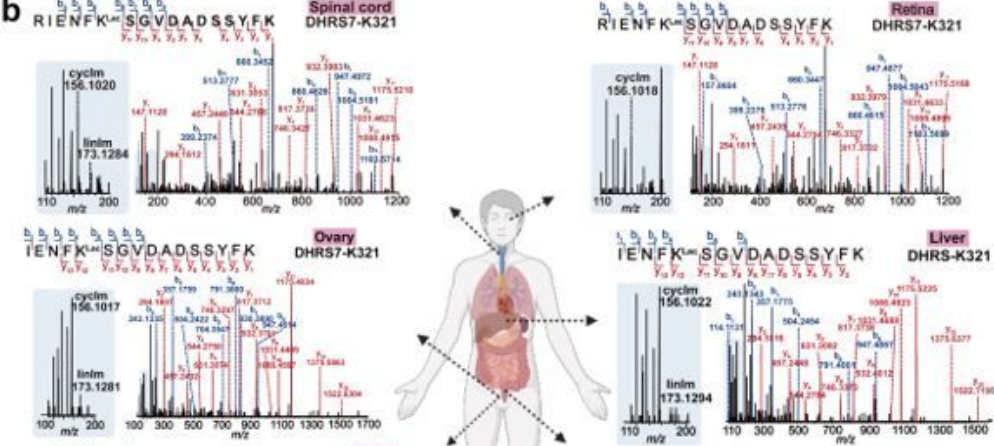

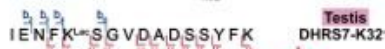

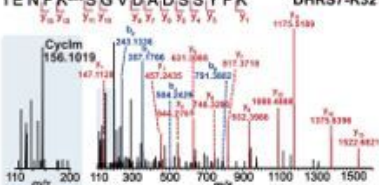

IENFKM SGVDADSSYFK DHRS-KM
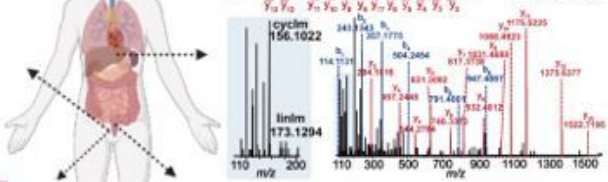

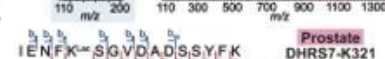

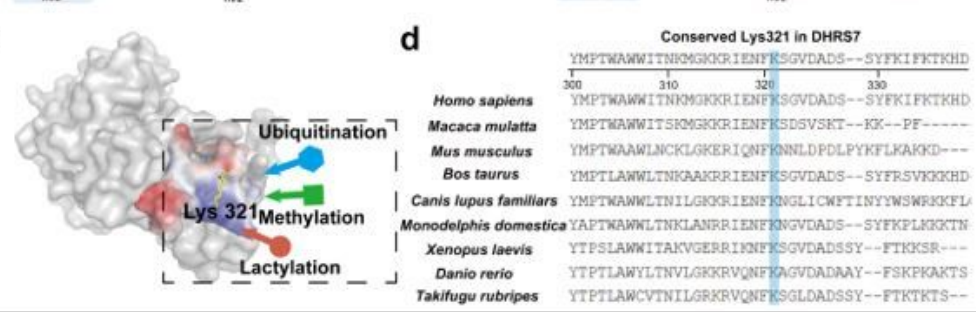

\section{Figure 4}

Mining lactylation from the draft map of the Human Proteome identifies widespread lactylation on DHRS7. (a) lon abundance of the cyclm ion in MS/MS spectra of lactylated peptides assigned by target-decoy database searching of proteomics data collected from six human tissue types. Each dot represents the relative ion abundance of the cyclm ion normalized to base peak in individual MS/MS spectrum of database searching-assigned lactylated peptides. (b) MS/MS spectra of lactylated K321-containing peptides of DHRS7 retrieved from the proteome shown in (a). (c) Protein model of DHRS7 predicted by AlphaFold. Yellow stick represents the K321 residue. Topological surface of DHRS7 is generated based on protein contact potential of vacuum electrostatics using PyMOL. PTM annotation is retrieved from iPTMnet. (d) Evolutionary conservation of K321 on DHRS7 across eukaryotic phylogeny. Proteomics data used in Fig. 4 was accessed through the ProteomeXchange Consortium with the dataset identifier PXD000561.

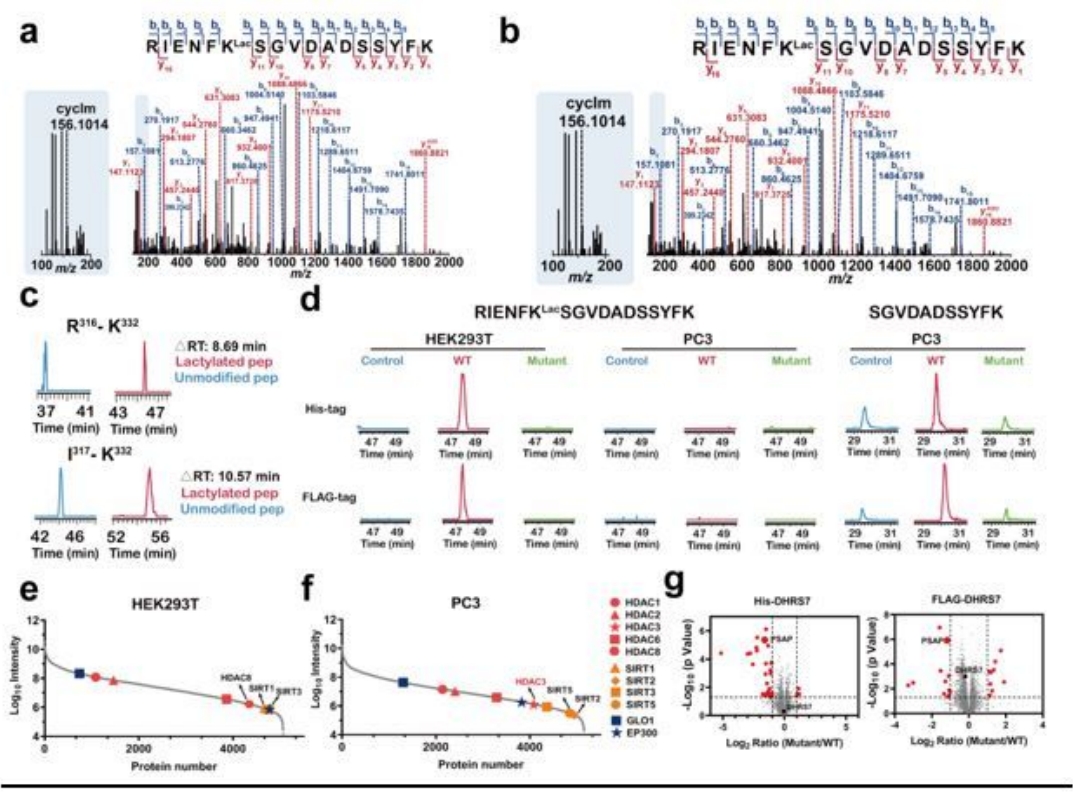




\section{Figure 5}

DHRS7 lactylation is cell type-specific and likely associated with delactylase abundance. (a-b) Sequence-related MS/MS ions and the cyclm ion together identified lactylated K321 on DHRS7 in DHRS7-overexpressed HEK293T cells. (c) Lactylation-conferred retention time shifts on K321-containing peptides. (d) Presence or absence of K321 lactylation in control vector, wild-type (WT) and K321A (mutant) DHRS7 plasmid-transfected HEK293T and PC3 cells are implied by XICs. (e-f) Protein abundance ranking of HEK293T (e) and PC3 cells (f). (g) Volcano plots showing global proteome abundances in response to site-directed mutagenesis of K321 on DHRS7 in HEK293T cells by shotgun proteomics ( $n=4 / g r o u p)$. Proteins displaying significantly altered abundance levels between the WT and mutant DHRS7-overexpressed 293T cells with the significance cutoff of $p<0.05$ and the fold change $>2$ or $<0.5$ are highlighted in red.

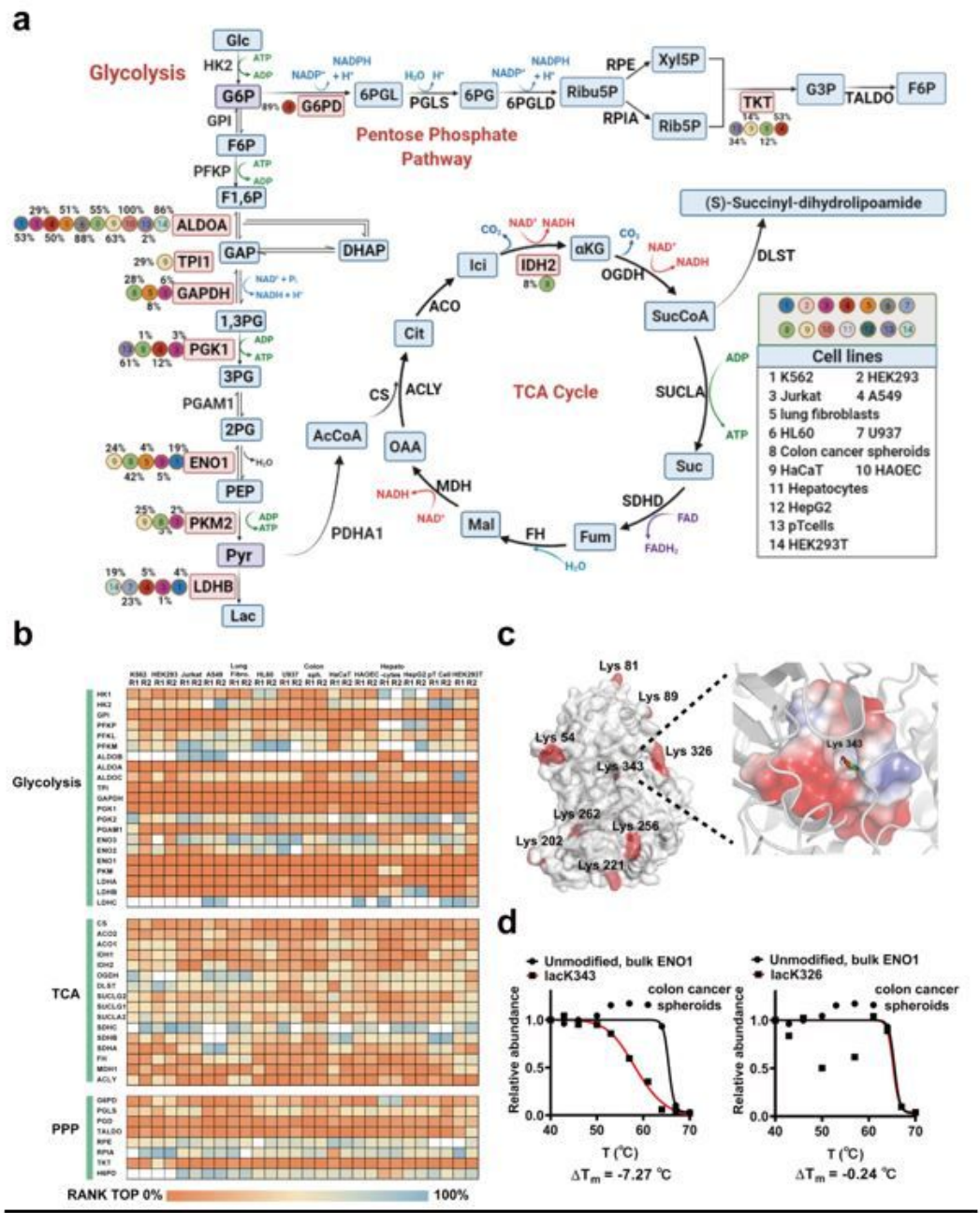

\section{Figure 6}

Mining the Meltome Atlas shows glycolytic enzymes are heavily lactylated in human cells and lactylation can alter thermal stability of carrier proteins. (a) A landscape of lactylation occurrence in enzymes involved in glycolysis, tricarboxylic acid (TCA) cycle, pentose phosphate pathway (PPP) in 14 human cell types. Relative ion abundance of the cyclm ion in corresponding MS/MS spectrum for lactylation assignment is labeled as \%. (b) Heatmap comparing protein abundance levels for the identified enzymes involved in glycolysis, TCA cycle, PPP as summarized in (a). (c) Crystal structure of human ENO1 with highlighted lactylation sites. Topological surface of ENO1 is generated based on protein contact potential of vacuum electrostatics using PyMOL. (d) Melting curves of the lactylated and non-lactylated ENO1 proteoforms in colon cancer spheroids. Proteomics data used in Fig. 6 was accessed through the ProteomeXchange Consortium with the dataset identifier PXD011929.

\section{Supplementary Files}

This is a list of supplementary files associated with this preprint. Click to download.

- Table1.SummaryofthelactylatedpeptidesidentifiedfromtherecombinantEN01proteindigestsusinganOrbitrapandSYNAPTG2SiQTOFinstrument.xIsx

- Table2.SummaryoftheidentifiedlactylatedpeptidesfromSILACprocessedandaffinityenrichedproteomedatasetscollectedfromMCF7cellsinresponsetorotenon

- Table3.SummaryofthelactylatedPSMsretrievedfromproteomedataofsixhumantissuetypesusingthreedifferentdatabasesearchengines.xlsx

- Table4.SummaryofthelactylatedPSMsidentifiedfromthe14humancelllinesavailableintheMeltomeAtlas.xlsx

- Supportinginformation.pdf 
- HuiYeEPCFlat.pdf

- HuiYeSI.pdf

Page 14/14 